\title{
As Atribuições de Causalidade no Ensino Fundamental: Relações com Variáveis Demográficas e Escolares
}

\author{
Natália Rodovalho Garcia \\ Evely Boruchovitch \\ Universidade Estadual de Campinas \\ Campinas, SP, Brasil
}

\begin{abstract}
RESUMO
Este artigo tem como objetivo identificar as atribuições de causalidade para sucesso e fracasso escolar, bem como verificar se existem relações entre essas atribuições e variáveis como sexo, repetência, idade e escolaridade. Participaram do estudo 275 alunos do $5^{\mathrm{O}}$ ao $9^{\mathrm{O}}$ ano de uma escola pública de Minas Gerais. A coleta de dados foi realizada por meio de um Questionário Demográfico e de uma Escala de Avaliação das Atribuições de Causalidade para Sucesso e Fracasso Escolar. Os dados foram analisados de acordo com os procedimentos da estatística descritiva e inferencial. A maioria dos participantes atribuiu a causas internas e controláveis os resultados escolares. Relações significantes entre as atribuições de causalidade e as variáveis demográficas e escolares foram encontradas. Os dados são discutidos em termos do importante papel da escola na promoção de atribuições causais compatíveis com a aprendizagem de qualidade.
\end{abstract}

Palavras-chave: Atribuições causais; Variáveis demográficas e escolares; Ensino fundamental.

\section{ABSTRACT \\ Causal Attributions in Elementary Education: Relations with Demographic and School Variables}

This article aimed to identify causal attributions for academic success and failure, as well as to verify whether there are relationships between these attributions and variables such as gender, grade repetition, age and education level. The study involved 275 students from 5th to 9th grade in a public school of Minas Gerais. Data collection was performed through a Demographic Questionnaire and a Rating Scale of Causal Attributions for Academic Success and Failure. Data were analyzed according to the procedures of descriptive and inferential statistics. Most participants attributed school results to internal and controllable causes. Significant relationships between causal attributions and demographic and school variables were found. The data are discussed in terms of the important role of schools in promoting causal attributions consistent with quality learning.

Keywords: Causal attributions; Demographic and school variables; Elementary education.

\section{RESUMEN}

\section{Las Atribuciones de Causalidad en la Educación Básica: Relaciones con Variables Demográficas y Escolares}

Este artículo tiene como objetivo identificar las atribuciones de causalidad para éxito y fracaso escolar, así como verificar se existen relaciones entre estas atribuciones y variables como sexo, reprobación, edad y escolaridad. Participaron del estudio 275 alumnos del $5^{\circ}$ al 9o año de una escuela pública de Minas Gerais. La recolección de datos fue realizada por medio de un Cuestionario Demográfico y de una Escala de Evaluación de las Atribuciones de Causalidad para Éxito y Fracaso Escolar. Los datos fueron analizados en conformidad con los procedimientos de la estadística descriptiva e inferencial. La mayoría de los participantes atribuyó las causas internas y controlables a los resultados escolares. Relaciones significantes entre las atribuciones de causalidad y las variables demográficas y escolares fueron encontradas. Los datos se analizan en términos de la importancia del papel de la escuela en la promoción de las atribuciones causales consistentes con un aprendizaje de calidad.

Palabras clave: Atribuciones causales; Variables demográficas y escolares; Educación básica. 


\section{INTRODUÇÃO}

Evidências mostram que o Brasil têm alcançado avanços importantes na área educacional, ao longo dos anos (IBGE, 2014). No entanto, é fato que o sistema escolar brasileiro ainda sofre de sérios problemas, entre eles a evasão escolar, o baixo nível de rendimento e a distorção entre idade e ano escolar dos estudantes (Boruchovitch, 1994; Soares \& Candian, 2007). Os indicadores sociais do IBGE (2014) apontam que entre 2001 e 2011, a pré-escola (educação para crianças de 4 a 5 anos) ampliou o seu atendimento em 11,3\% e o acesso ao ensino fundamental está praticamente universalizado, atendendo a $98,2 \%$ dos alunos em idade escolar. Todavia, a porcentagem de concluintes ainda está distante da universalização, sendo fundamental compreender os fatores intervenientes no desempenho escolar, de modo a garantir condições aos alunos de progredirem, normalmente, no sistema educacional.

De acordo com Patto (1990), diversas teorias foram elaboradas com o objetivo de explicar o fracasso escolar brasileiro. Em linhas gerais, como mencionam estudiosos do tema (Leite, 1988; Neves \& Almeida, 1996; Pain, 1985; Ribeiro, 1993), algumas teorias dominantes atribuem a qualidade do desempenho escolar a características biológicas, psicológicas e sociais do aluno, e aos fatores do contexto de aprendizagem como as características do professor. Outras o relacionam às condições políticas, sociais, econômicas e culturais que intervém no contexto educacional e sobre o processo de ensino e aprendizagem. As explicações tradicionais do fracasso escolar ora focalizavam unicamente as características individuais, ora as condições do ambiente, não levando em conta toda a complexidade do processo de escolarização. Constata-se, entretanto, que profissionais da área educacional vêm se esforçando na tentativa de superar as explicações tradicionais, considerando, simultaneamente, tanto os fatores internos quanto os externos na complexa análise do processo de ensinoaprendizagem (Collares, 1995; Leite, 1988; Neves \& Almeida, 1996; Patto, 1990; Souza, 2009).

Pesquisadores tem buscado uma compreensão maior da relação existente entre as crenças individuais e o comportamento das pessoas em contextos de realização. Nessa direção, a Teoria da Atribuição de Causalidade revelou-se valiosa e alvo de estudos sistemáticos em diversos contextos, sobretudo o educativo (Almeida, Miranda, Salgado, Silva, \& Martins, 2012; Ganda \& Boruchovitch, 2012; Janeiro, 2011; Marques \& Dela Coleta, 2010; Sá, Dela Coleta, \& Dela Coleta, 2011; Timm, Argimon, \& Wendt, 2011, Weiner, 1972, 2004, 2013).
Elaborada por Weiner (1972), a Teoria da Atribuição de Causalidade tem sua base na Psicologia Cognitiva da Motivação e centra-se na análise de cognições específicas do sujeito como as atribuições para sucesso e fracasso em situações de realização (Barreira, 2010). As atribuições de causalidade referem-se à tendência do ser humano a atribuir causas aos eventos em geral, sendo esse um processo que auxilia o indivíduo a entender melhor o ambiente em que vive e a se adaptar às exigências do meio. Essas atribuições dizem respeito às interpretações que os indivíduos fazem das causas dos fatos, dos comportamentos de outros e também de si próprio. Desempenham um papel fundamental na explicação da ação futura, da motivação e da emoção (Martini \& Boruchovitch, 2004; Weiner, 1972, 1991, 2004; 2010). Pode-se dizer que a maneira como a pessoa percebe a causa do ato, independente da realidade objetiva e dependente das interferências cognitivas vai determinar o comportamento subsequente, bem como os seus sentimentos.

Weiner $(1972,2004,2010)$ enfocou as relações entre atribuições de causalidade e o sucesso e fracasso no desempenho de tarefas no contexto educativo. Neste contexto, as atribuições causais, dizem respeito às interpretações individuais que os alunos fazem sobre os resultados escolares e acadêmicos obtidos. Apresentam relações com as expectativas do aluno para as atividades e, por conseguinte, interferem na motivação e no desempenho futuro (Dela Coleta \& Dela Coleta, 2006; Ferreira et al., 2002; Martini \& Boruchovitch, 2004). De acordo com Weiner (1985), há diversas causas possíveis para explicar o sucesso e o fracasso em uma tarefa, como por exemplo, prestar atenção, esforço, capacidade, facilidade da tarefa, ajuda do professor e sorte. Assim, o autor elaborou o modelo tridimensional de classificação das causas; cada uma das três dimensões tem dois polos distintos: localidade, estabilidade e controlabilidade. A localidade refere-se à localização da causa, que pode estar no próprio indivíduo (interna) ou na situação (externa). A estabilidade diz respeito à natureza temporal da causa, ao fato de ela perdurar no tempo (estável) ou não (instável). Por fim, a controlabilidade concerne à influência que pode ser exercida sobre a causa (controlável) ou não (incontrolável). A classificação de uma atribuição nas dimensões da causalidade depende do significado subjetivo que a causa possua para o indivíduo. Nesse sentido, a mesma causa pode ser interpretada, de maneira diferente, por diversas pessoas (Martini \& Boruchovitch, 2004; Weiner, 2004).

Em termos de consequências, a interpretação que o sujeito faz sobre as causas de um determinado evento está associada à motivação, à realização, à orientação 
da tarefa, à expectativa de sucesso e fracasso futuros e as reações emocionais dos estudantes (Boruchovitch \& Martini, 1997). Pesquisadores verificaram que as atribuições de causalidade são mais compatíveis com a motivação para aprender, quando o sujeito atribui causas internas e controláveis ao desempenho escolar obtido como, por exemplo, o esforço. Isso ocorre porque o esforço pode ser instrumentalizado pelo sujeito, tanto para manter ou aumentar o sucesso, como para evitar futuros fracassos futuros (Martini \& Boruchovitch, 2004; Weiner, 1972, 2004; 2010). A atribuição de sucessos a causas externas e incontroláveis, como a sorte, e também o fracasso a causas externas e/ou internas, incontroláveis, como dificuldade da tarefa e a falta de capacidade, seriam mais desfavoráveis por gerar uma percepção de ausência de controle, tanto para produzir o êxito, como para evitar o fracasso (Martini \& Boruchovitch, 2004; Weiner, 1972, 2004).

Devido à importância da Teoria de Atribuição de Causalidade no campo educacional, pesquisas têm sido conduzidas com o intuito de conhecer as atribuições de causalidade em estudantes nos mais diversos níveis de escolaridade e suas relações com variáveis demográficas e escolares como o gênero, a idade, o desempenho, a escolaridade, entre outras (Almeida, Miranda, \& Guisande, 2008; Jung, 2008; Miranda, Almeida, Boruchovitch, Almeida, \& Abreu, 2012; Paiva \& Boruchovitch, 2010; Sahinkarakas, 2011; Stephanou, 2012).

De um modo geral, os estudos internacionais e nacionais que buscaram identificar as atribuições de causalidade para o sucesso e para o fracasso escolar em alunos dos Ensinos Fundamental e Médio verificaram que os estudantes, em sua maioria, atribuem a si mesmos a responsabilidade pelo resultado escolar obtido, seja sucesso ou fracasso (Almeida, Miranda, \& Guisande, 2008; Ferreira et al., 2002; Martini, 2003; Martini \& Boruchovitch, 2009; Miranda et al., 2012; Neves \& Faria, 2007; Sahinkarakas, 2011; Yoshimoto, 2004). Além disso, pesquisas revelaram que variáveis como sexo, idade, desempenho escolar e histórico de reprovação exercem impacto nas atribuições de causalidade dos estudantes (Dela Coleta \& Dela Coleta, 2006; Khodayarifard, Anshel, \& Brinthaupt, 2006; Martini \& Boruchovitch, 2004; Martini \& Del Prette, 2005; Miranda et al., 2012).

Verifica-se que os meninos parecem atribuir o êxito à capacidade e a meninas atribuem mais o seu alto rendimento ao esforço, as estratégias de estudo ou, então, a fatores externos. Por outro lado, na explicação do baixo desempenho, os alunos do sexo masculino parecem recorrer mais a causas externas, já os do feminino mencionam mais a falta de esforço e de capacidade (Boruchovitch, 1994; Martini, 2003; Martini \& Boruchovitch, 2004, 2009; Martini \& Del Prette, 2005; Schilieper, 2001). Para Martini e Boruchovitch (2004) as diferenças nas atribuições de causalidade em função do sexo dos alunos, podem ser decorrentes das formas singulares que cada um dos sexos tem de extrair as informações do ambiente.

Segundo alguns estudos realizados na área, com o avançar da idade e da escolaridade, os alunos tendem a mencionar mais causas internas como fatores responsáveis do sucesso e do fracasso alcançado (Boruchovitch, 2004; Khodayarifard et al., 2006). Isto ocorre porque à medida que avançam na idade e na escolaridade, os alunos tornam-se mais propensos a perceber que o sucesso e o fracasso estão relacionados ao nível de esforço despendido e não à falta de sorte, de capacidade ou de afeto por parte do professor (Boruchovitch, 2004; Martini \& Boruchovitch, 2004; Miranda et al., 2012). O esperado é que, a partir das experiências vividas na escola e da intervenção adequada de adultos e profissionais nos momentos de sucesso e de fracasso, o aluno gradualmente aprenda que o bom ou mau desempenho depende mais de si próprio do que da ação de forças externas a ele (Boruchovitch, 2001, 2004; Dela Coleta \& Dela Coleta, 2006; Martini $\&$ Boruchovitch, 2004; Miranda et al., 2012).

No que concerne a variável repetência, Almeida e Guisande (2010) apontam que os alunos fragilizados em termos de rendimento acadêmico tendem a atribuir os seus eventuais sucessos a fatores que lhes são externos, e os seus insucessos, a variáveis internas, geralmente a falta de capacidade. Esse é um padrão de atribuições pouco adaptativo, por levar o aluno a duvidar das próprias capacidades para melhorar o seu desempenho e a considerar os seus esforços inúteis. Como consequência, emergem sentimentos de frustração, de desmotivação e de baixa autoestima (Almeida \& Guisande, 2010; Martini \& Boruchovitch, 2004).

A atribuição de causalidade do aluno para o desempenho escolar obtido tem um impacto no êxito e na qualidade de sua aprendizagem (Martini \& Boruchovitch, 2004; Weiner, 1979, 2010). Assim, investigar este construto em estudantes é importante para o aprofundamento teórico na área, o aperfeiçoamento da prática docente e a elaboração de programas de ação e intervenção no contexto educativo. Analisar as possíveis diferenças nas atribuições de causalidade para o sucesso e para o fracasso escolar em função de variáveis demográficas e escolares é também relevante, pois pode auxiliar os profissionais da Educação, especialmente, os docentes quanto ao modo de lidar com cada grupo, atendendo às suas 
necessidades específicas. Ademais, como a maior parte dos estudos nacionais sobre o tema foi realizada em cidades grandes e teve por base instrumentos qualitativos (Martini \& Del Prette, 2005; Paiva \& Boruchovitch, 2010; Yoshimoto, 2004), o presente trabalho tem como objetivo identificar as atribuições de causalidade para sucesso e fracasso escolar, bem como verificar a existência de relações entre essas atribuições causais e variáveis como sexo, repetência, idade e escolaridade, entre estudantes de uma realidade pouco explorada no interior do estado de Minas Gerais por meio de um instrumento quantitativo. Realidades como essa precisa ser mais conhecida e foco de atenção de pesquisadores.

\section{MÉTODO}

O presente estudo constituiu em uma pesquisa quantitativa de natureza exploratória, desenvolvida no ano de 2012 em uma escola pública localizada, em um bairro pobre de uma cidade de pequeno porte do interior do estado de Minas Gerais. À época da realização da pesquisa, a escola atendia somente o ensino fundamental, com aproximadamente 600 alunos distribuídos nos turnos matutino, vespertino e noturno. A equipe pedagógica era formada por 26 professores, uma diretora, uma vice-diretora e uma supervisora. A escola atende uma clientela de nível socioeconômico desfavorecido. Os estudantes são, em geral, filhos de trabalhadores rurais e da indústria cerâmica. A escolha do município e da escola se deu pela relevância de se estudar contextos educacionais desfavorecidos, ainda muito pouco investigados.

\section{Participantes}

A amostra foi composta por 275 alunos do $2^{\mathrm{O}}$ ciclo do Ensino Fundamental da referida escola. Dos participantes, 50,18\% $(\mathrm{n}=138)$ eram do sexo masculino e $49,82 \%(n=137)$ do sexo feminino. A idade variou entre 9 e 16 anos, com média de idades de 11,96 $(\mathrm{DP}=1,59)$. Quanto à escolaridade, 28,73\% alunos $(\mathrm{n}=79)$ frequentavam o $5^{\circ}$ ano escolar, $26,91 \%(\mathrm{n}=74)$ o $6^{\mathrm{o}}$ ano, $19,64 \%(\mathrm{n}=54)$ o $7^{\mathrm{o}}$ ano, $14,18 \%(\mathrm{n}=39)$ o $8^{\circ}$ ano e $10,55 \%(n=29)$ estavam no 9 o ano. A maioria dos estudantes, 65,09\% $(\mathrm{n}=179)$, não apresentavam repetência escolar e a minoria, 34,91\% $(n=96)$ já havia repetido pelo menos um ano. Escolheu-se trabalhar com alunos a partir do $5^{\mathrm{o}}$ ano escolar, por ser um período no qual os indivíduos completam o processo de alfabetização e vivenciam mudanças no sistema educacional que exigem do aluno uma reorganização dos hábitos escolares. Decidiu-se, também, por todos os anos do $2^{\mathrm{o}}$ ciclo do Ensino Fundamental, com o objetivo de verificar se existem diferenças na atribuição de causalidade dos alunos, com o avançar da idade e do ano escolar.

\section{Instrumentos}

Foram utilizados dois instrumentos para a coleta de dados:

- Questionário demográfico: esse instrumento foi elaborado para o presente estudo com o objetivo de coletar informações referentes às variáveis como sexo, repetência escolar, idade e escolaridade.

- Escala de Avaliação das Atribuições de Causalidade para Sucesso e Fracasso Escolar: essa escala foi desenvolvida por Boruchovitch e Santos (2011). Teve como base para a sua construção os fundamentos da Teoria da Atribuição de Causalidade de Weiner (1972), descrita na introdução. Visa avaliar quais as causas que os alunos atribuem como possíveis explicações para o seu sucesso e fracasso escolar. O instrumento é composto por duas subescalas, atribuição frente ao sucesso e atribuição frente ao fracasso. Possui 55 itens com três alternativas de escolha: sempre, às vezes e nunca. No que se referem à pontuação, três pontos são atribuídos para a opção "sempre", dois pontos para "às vezes" e um ponto para "nunca". Os itens são classificados de acordo com a situação (sucesso ou fracasso) e as dimensões (localidade ou controlabilidade). Assim, os itens 01, 02, 05, 06, 09, $10,13,14,15,18,19,21,22,25,26,27,30$ e 31 avaliam se o aluno atribui o sucesso a causas internas ou externas (Exemplos: "Tiro boas notas porque sou inteligente."; "Tiro notas boas porque tenho o apoio da minha família.") Os itens 34, 35, 36, 39, 40, 41, 44, 45, $46,49,50,51,54$ e 55 medem se o estudante atribui o fracasso a fatores internos ou externos (Exemplos: "Vou mal na escola porque não presto atenção na aula."; "Tiro notas baixas porque não recebo apoio da minha família."). Os itens 03, 04, 07, 08, 11, 12, 16, $17,20,23,24,28$ e 29 avaliam se o estudante atribui o sucesso a fatores controláveis ou incontroláveis (Exemplo: "Consigo deixar de ser bagunceiro na hora que eu quiser."). Os itens $32,33,37,38,42,43,47,48$, 52 e 53 medem se o aluno atribui o fracasso a causas controláveis ou incontroláveis (Exemplo: "Não consigo fazer nada para ser mais inteligente."). O escore total pode variar de 55 a 165 pontos. Quanto mais alto, maior a tendência do aluno a fazer atribuições causais internas e controláveis. Para o cálculo dos escores, devem-se inverter os valores das opções de resposta dos seguintes itens $05,06,10,13,14,18,19,21,25$, $32,33,34,36,37,38,39,41,42,43,46,47,48,49$, $50,52,53$ e 54 , por se relacionarem a causas externas e incontroláveis. Esse instrumento foi utilizado pela 
primeira vez nesta presente pesquisa. Encontram-se em andamento, pelos autores da escala, estudos referentes a sua validade de construto. A consistência interna da escala foi estimada pelo Alpha de Cronbach tendo-se obtidos valores aceitáveis, de acordo com os critérios de Prieto e Muñiz (2000), tanto para a escala total $(\alpha=0,70)$ quanto para as subescalas sucesso $(\alpha=0,64)$ e fracasso $(\alpha=0,64)$.

\section{Procedimentos}

A presente pesquisa cumpriu as exigências da Resolução n. 196/96 do Conselho Nacional de Saúde (CNS), de modo que a coleta de dados foi realizada somente após a aprovação do Comitê de Ética em Pesquisa da Faculdade de Ciências Médicas da UNICAMP (Parecer n. 1248/2011, CAAE: 1151.0.146.146-11).

Antes de realizar a coleta de dados propriamente dita, foi feito um estudo-piloto com o propósito de aprimorar os instrumentos de pesquisa e os métodos de aplicação. Participaram desse estudo-piloto 10 alunos, regularmente matriculados no $5^{\circ}$, no $6^{\circ}$, no $7^{\circ}$, no $8^{\circ}$ e no $9^{\circ}$ anos escolares, sendo dois de cada ano, da mesma escola em que a coleta definitiva ocorreu, mas esses alunos não foram integrantes da amostra total da pesquisa. A partir desse procedimento, fez-se necessário alterar a diagramação dos instrumentos, deixando-os em um formato mais fácil de preenchimento, em que todas as opções de resposta são apresentadas após cada item. Esse modelo de organização do questionário e da escala foi construído com a ajuda de uma pedagoga, mestranda em Psicologia Educacional. O enunciado da escala foi ligeiramente alterado e padronizado para deixar claro que, ao responder o instrumento, os alunos deveriam assinalar com um $\mathrm{X}$ a alternativa correspondente à sua resposta.

Posteriormente, os alunos do $5^{\mathrm{O}}$ ao $9^{\mathrm{O}}$ ano escolar foram convidados a participar da pesquisa e foilhes entregue o termo de consentimento por escrito, direcionado aos pais, com o objetivo de proporcionar um amplo esclarecimento sobre o trabalho e solicitar a autorização deles para a participação de seus filhos(as). Foi assegurada aos pais a participação voluntária, bem como o caráter confidencial da pesquisa e que ela não afetaria o desempenho do estudante na escola.

A coleta definitiva dos dados foi realizada com agendamento prévio e no horário de melhor conveniência para os participantes e para a instituição. Foram formados grupos de seis a oito alunos do mesmo ano escolar. Entretanto, é importante ressaltar que os alunos responderam individualmente os instrumentos. Diante da devolutiva do termo de consentimento assinado pelos pais ou responsáveis, a pesquisadora chamava os alunos em sala de aula e os direcionava para uma sala da escola reservada para o trabalho. O ambiente foi organizado com carteiras individuais, contendo lápis e borracha. Antes da aplicação dos instrumentos, a pesquisadora instruía os alunos sobre o seu preenchimento e, no momento de resposta aos itens, realizava a leitura em voz alta de cada afirmativa para eles os responderem individualmente, em silêncio. Cada grupo levou, aproximadamente, 60 minutos para completar todos os instrumentos.

As análises estatísticas foram realizadas com recurso ao programa estatístico SAS System for Windows, versão 9.2. Os dados provenientes da Escala de Avaliação das Atribuições de Causalidade para Sucesso e Fracasso Escolar foram estudados em termos da estatística descritiva e inferencial. Foi utilizada a estatística descritiva com o objetivo de sintetizar os resultados da amostra, por meio de valores de média, desvio padrão, pontuação mínima e máxima. A análise inferencial dos dados teve o intuito de explorar relações entre resultados na Escala de Avaliação das Atribuições de Causalidade para Sucesso e Fracasso Escolar e as variáveis demográficas como sexo, repetência, idade e escolaridade. Mais precisamente, para a comparação do escore da escala e as variáveis demográficas foi utilizado o Teste de Mann-Whitney para dois grupos e o Teste de Kruskal-Wallis para três ou mais grupos. As correlações entre as variáveis numéricas como a idade e os escores da escala de atribuição de causalidade foram também estimadas por meio do coeficiente de correlação de Spearman, devido à ausência de distribuição normal na amostra, constatada pelo Teste de Shapiro-Wilk e, interpretadas de acordo com Levin e Fox (2004). O nível de significância adotado para os testes estatísticos foi de $5 \%$, ou seja, $p<0,05$.

\section{RESULTADOS}

A Tabela 1 apresenta a pontuação dos participantes nas subescalas e na escala de atribuição de causalidade, bem como a média, o desvio padrão e valores mínimos e máximos obtidos. Em termos gerais, pode-se inferir que os alunos tendem a apresentar como justificativas para o seu desempenho escolar causas internas e controláveis, se responsabilizando tanto pelo bom como pelo fraco desempenho escolar.

Resultados provenientes da análise comparativa entre a escala de atribuição de causalidade e o sexo, bem como da comparação entre a escala de atribuição de causalidade e a repetência podem ser vistos na Tabela 2. Pode-se notar que não foram encontradas diferenças estatisticamente significantes quanto ao sexo nas pontuações das subescalas e totais da 


\section{TABELA 1}

Média, desvio padrão e pontuação mínima e máxima dos estudantes na escala de atribuição de causalidade

\begin{tabular}{lccccc}
\hline & $N$ & Média & Desvio Padrão & Mínimo & Máximo \\
\hline Atribuição frente ao Sucesso & 273 & 68,31 & 5,35 & 53,00 & 81,00 \\
Atribuição frente ao Fracasso & 273 & 54,64 & 5,13 & 35,00 & 64,00 \\
Atribuição de Causalidade Total & 271 & 123,0 & 8,19 & 92,00 & 143,0 \\
\hline
\end{tabular}

escala de atribuição de causalidade, indicando que tanto os meninos como as meninas fazem atribuições semelhantes ao desempenho escolar obtido. Já em relação à repetência, nota-se que há diferenças significantes entre os escores na subescala "atribuição frente ao sucesso" e na escala "atribuição de causalidade total" e essa variável. Os dados parecem indicar que, para os alunos sem repetência, os resultados e sucessos escolares alcançados decorrem, em sua maior parte, de fatores internos e controláveis. Isso não é observado nos alunos que já repetiram pelo menos um ano escolar. Quanto à subescala "atribuição frente ao fracasso" não foi encontrada diferença significante com a repetência escolar.

A Tabela 3 apresenta análises comparativas entre a escala de atribuição de causalidade e a idade e, entre esse instrumento e a escolaridade. No que se referem à idade, os participantes foram organizados em quatro grupos: estudantes com idade entre 9 e 10 anos $(n=55)$, 11 e 12 anos $(n=120), 13$ e 14 anos $(n=85)$ e entre 15 e 16 anos de idade $(n=15)$. Observa-se que há diferenças altamente significantes nos escores apresentados pela amostra na subescala "atribuição frente ao sucesso" em função da idade. Nessa subescala, as maiores pontuações foram obtidas pelos alunos com idade entre 9 e 10 anos. Esses dados sugerem que os alunos mais novos tendem a acreditar mais que o sucesso escolar seja desencadeado por fatores internos e controláveis.
Nos escores da subescala "atribuição frente ao fracasso" e da escala "atribuição de causalidade total" não foram encontradas diferenças significativas no que diz respeito à idade. Além disso, como mostra a Tabela 4 , verifica-se correlação altamente significante, negativa e fraca da idade com escore de atribuição frente ao sucesso e atribuição de causalidade total $(\mathrm{r}=-0,25378$ e $p<0,0001 ; r=-0,14588$ e $p=0,0163$ ). Dessa forma, pode-se sugerir que quanto mais velho é o participante, menos ele atribui às causas dos resultados e sucessos escolares a fatores internos e controláveis.

Em relação à escolaridade, os dados revelam que há diferenças estatisticamente significantes entre os escores obtidos pelos alunos na subescala "atribuição frente ao sucesso" e o ano escolar. Dessa forma, podese supor que os estudantes do $5^{\circ}$ ano de escolaridade atribuem o sucesso escolar mais a causas de ordem interna e controlável, quando comparados aos demais anos escolares. Quanto aos escores da subescala "atribuição frente ao fracasso" e da escala "atribuição de causalidade total" não foram observadas diferenças significantes em relação ao ano escolar dos alunos.

\section{DISCUSSÃO}

O objetivo do presente estudo foi identificar as atribuições de causalidade para sucesso e fracasso escolar, bem como verificar se existem relações entre

TABELA 2

Comparação dos escores da escala de atribuição de causalidade dos estudantes com o sexo e a repetência

\begin{tabular}{|c|c|c|c|c|c|c|}
\hline & \multicolumn{3}{|c|}{ Sexo } & \multicolumn{3}{|c|}{ Repetência } \\
\hline & \multirow{2}{*}{$\begin{array}{c}\text { Masculino } \\
\text { Média (D.P.) }\end{array}$} & \multirow{2}{*}{$\begin{array}{c}\text { Feminino } \\
\text { Média (D.P.) }\end{array}$} & \multicolumn{2}{|c|}{ Sim } & \multicolumn{2}{|c|}{ Não } \\
\hline & & & Valor-p & Média (D.P.) & Média (D.P.) & Valor-p \\
\hline Atribuição frente ao Sucesso & $67,88(5,38)$ & $68,74(5,31)$ & $\mathrm{p}=0,250$ & $66,35(4,91)$ & $69,37(5,29)$ & $\mathrm{p}<0,001^{*}$ \\
\hline Atribuição frente ao Fracasso & $54,80(5,07)$ & $54,49(5,20)$ & $\mathrm{p}=0,705$ & $54,64(5,23)$ & $54,65(5,09)$ & $\mathrm{p}=0,806$ \\
\hline Atribuição de Causalidade Total & $122,71(7,93)$ & $123,20(8,46)$ & $\mathrm{p}=0,601$ & $120,99(7,44)$ & $124,03(8,40)$ & $\mathrm{p}=0,002 *$ \\
\hline
\end{tabular}

Nota: Valor-p referente ao Teste de Mann-Whitney para comparação das variáveis entre 2 grupos. $* \mathrm{p}<0,05$ 
TABELA 3

Comparação dos escores da escala de atribuição de causalidade dos estudantes com a faixa etária e o ano escolar

\begin{tabular}{|c|c|c|c|c|c|c|c|c|c|c|c|}
\hline \multirow{3}{*}{ Atribuição } & \multicolumn{5}{|c|}{ Idade } & \multicolumn{6}{|c|}{ Ano Escolar } \\
\hline & $09-10$ & $11-12$ & $13-14$ & $15-16$ & & $5^{\mathrm{o}}$ & $6^{\mathrm{o}}$ & $7^{\circ}$ & $8^{\mathrm{o}}$ & $9^{\underline{o}}$ & \\
\hline & $\begin{array}{c}\text { Média } \\
(D P)\end{array}$ & $\begin{array}{c}\text { Média } \\
(D P)\end{array}$ & $\begin{array}{c}\text { Média } \\
(D P)\end{array}$ & $\begin{array}{c}\text { Média } \\
(D P)\end{array}$ & Valor-p & $\begin{array}{c}\text { Média } \\
(D P)\end{array}$ & $\begin{array}{c}\text { Média } \\
(D P)\end{array}$ & $\begin{array}{c}\text { Média } \\
(D P)\end{array}$ & $\begin{array}{c}\text { Média } \\
(D P)\end{array}$ & $\begin{array}{c}\text { Média } \\
(D P)\end{array}$ & Valor- $p$ \\
\hline $\begin{array}{l}\text { Atribuição } \\
\text { frente ao } \\
\text { Sucesso }\end{array}$ & $\begin{array}{c}70,74 \\
(5,11)\end{array}$ & $\begin{array}{l}68,18 \\
(5,31)\end{array}$ & $\begin{array}{l}67,22 \\
(5,31)\end{array}$ & $\begin{array}{l}66,93 \\
(4,37)\end{array}$ & $0,001 *$ & $\begin{array}{l}69,66 \\
(5,45)\end{array}$ & $\begin{array}{l}68,64 \\
(5,08)\end{array}$ & $\begin{array}{l}66,96 \\
(5,27)\end{array}$ & $\begin{array}{l}67,36 \\
(5,55)\end{array}$ & $\begin{array}{l}67,66 \\
(5,04)\end{array}$ & $0,041^{*}$ \\
\hline $\begin{array}{l}\text { Atribuição } \\
\text { frente ao } \\
\text { Fracasso }\end{array}$ & $\begin{array}{l}55,05 \\
(4,76)\end{array}$ & $\begin{array}{l}54,26 \\
(4,89)\end{array}$ & $\begin{array}{l}54,75 \\
(5,73)\end{array}$ & $\begin{array}{l}55,60 \\
(4,97)\end{array}$ & 0,630 & $\begin{array}{l}53,53 \\
(5,93)\end{array}$ & $\begin{array}{l}54,91 \\
(3,97)\end{array}$ & $\begin{array}{l}55,44 \\
(5,15)\end{array}$ & $\begin{array}{l}55,00 \\
(5,05)\end{array}$ & $\begin{array}{l}55,03 \\
(5,37)\end{array}$ & 0,453 \\
\hline $\begin{array}{l}\text { Atribuição de } \\
\text { Causalidade } \\
\text { Total }\end{array}$ & $\begin{array}{c}125,70 \\
(8,30)\end{array}$ & $\begin{array}{c}122,44 \\
(7,69)\end{array}$ & $\begin{array}{c}122,02 \\
(8,59)\end{array}$ & $\begin{array}{c}122,53 \\
(8,06)\end{array}$ & 0,063 & $\begin{array}{l}123,11 \\
(9,02)\end{array}$ & $\begin{array}{c}123,54 \\
(6,91)\end{array}$ & $\begin{array}{c}122,41 \\
(8,66)\end{array}$ & $\begin{array}{c}122,47 \\
(8,34)\end{array}$ & $\begin{array}{l}122,69 \\
(8,28)\end{array}$ & 0,977 \\
\hline
\end{tabular}

Nota: Valor- $p$ referente ao Teste de Kruskal-Wallis para comparação das variáveis entre 3 grupos. $* p<0,05$

TABELA 4

Correlações da idade com os escores da escala de atribuição de causalidade dos estudantes

\begin{tabular}{lcll}
\hline \multicolumn{1}{c}{ Atribuição } & \multicolumn{1}{c}{$r$} & \multicolumn{1}{c}{$p$} & $n$ \\
\hline Atribuição frente ao Sucesso & $-0,25378$ & $<0,0001$ & 273 \\
Atribuição frente ao Fracasso & 0,01885 & 0,7565 & 273 \\
Atribuição de Causalidade Total & $-0,14588$ & 0,0163 & 271 \\
\hline
\end{tabular}

$\mathrm{r}=$ coeficiente de correlação de Spearman; $p=$ Valor- $p ; \mathrm{n}=$ número de sujeitos.

essas atribuições e variáveis como sexo, repetência, idade e escolaridade. No que diz respeito às atribuições de causalidade para o sucesso e o fracasso escolar, os dados obtidos nesta amostra estão em consonância com os resultados de pesquisas anteriores, pois se observa o predomínio de atribuições causais internas e controláveis tanto para o sucesso como para o fracasso escolar (Ferreira et al., 2002; Boruchovitch, 2004; Martini \& Boruchovitch, 2009; Martini \& Del Prette, 2005). Esses resultados, por serem provenientes de estudos nacionais oferecem plausibilidade à expectativa de que estudantes brasileiros dos Ensinos Fundamental e Médio parecem relatar uma orientação atribucional adequada à motivação para aprender. Nas situações escolares os alunos com atribuições de causalidade mais internas e controláveis tendem a apresentar melhor desempenho e maior dedicação, já que a internalidade e a controlabilidade das causas favorecem a percepção de que é possível aprimorar características pessoais e superar desempenhos malsucedidos. Esse padrão atribucional contribui para a aprendizagem autorregulada, na qual o indivíduo acredita ser capaz de orientar suas cognições e comportamentos em direção à realização de seus objetivos (Cleary \& Zimmerman, 2012).

Entretanto, dados controversos foram encontrados nas pesquisas de Soric (2009) e de Russell (1982) sobre as atribuições de causalidade para o desempenho escolar de estudantes da Educação Básica de escolas da Croácia e americanas respectivamente, no qual alunos atribuíram o sucesso escolar a causas controláveis e o fracasso a causas incontroláveis. Dessa forma, ressaltase a necessidade de futuras pesquisas a fim de verificar possíveis intervenientes nas atribuições causais em função da nacionalidade dos estudantes.

No que concerne à existência de diferenças nos escores da escala de atribuição de causalidade e a variável sexo, não foram encontradas diferenças estatisticamente significantes nos escores das subescalas e totais da escala de atribuição, sugerindo que os meninos e as meninas fazem atribuições causais ao desempenho escolar de forma semelhante. Destacase que esta amostra foi equilibrada quanto ao sexo, sendo composta por 138 alunos do sexo masculino e 137 do feminino. Resultados parecidos foram obtidos na pesquisa de Mascarenhas, Almeida e Barca (2005) em uma amostra formada por estudantes de escolas públicas do Estado de Rondônia, de ambos os sexos, com idade entre 13 e 19 anos. Fatemi e Asghari (2012) também não encontraram diferenças de sexo nas atribuições causais de alunos iranianos para os resultados alcançados na aprendizagem do Inglês como uma segunda língua. No entanto, evidências provenientes da Teoria da Atribuição de Causalidade e também de muitos estudos localizados na revisão de 
literatura (Boruchovitch, 1994; Martini \& Del Prette, 2005; Martini \& Boruchovitch, 2004, 2009; Schilieper, 2001) revelam a existência de diferenças sexuais nas atribuições causais de sucesso e de fracasso no contexto escolar. Conforme exposto anteriormente, em situações de sucesso, os meninos parecem atribuir o êxito à capacidade e a meninas atribuem mais ao esforço. Nos casos de baixo desempenho, os meninos fazem mais atribuições causais a fatores externos, já as meninas mencionam mais fatores internos (Boruchovitch, 1994; Martini \& Del Prette, 2005; Martini \& Boruchovitch, 2004, 2009; Schilieper, 2001). Em virtude das diferenças sexuais encontradas nas atribuições causais de escolares em algumas pesquisas e não em outras e a relevância da variável sexo no contexto educativo, recomenda-se que novos estudos sejam conduzidos com essa variável.

Ao analisar a atribuição de causalidade em função da repetência, os dados corroboram os achados de pesquisas anteriores, nas quais os alunos sem reprovação atribuíram o sucesso e o desempenho escolar obtido mais a fatores internos e controláveis quando comparados aos estudantes com histórico de repetência. (Almeida \& Guisande, 2010; Boruchovitch, 2004; Silva, Mascarenhas, \& Silva, 2011). Ressalta-se que os alunos com histórico de repetência merecem maior atenção dos professores no sentido de ajudá-los a perceber como o maior ou o menor esforço e ou o uso de estratégias de aprendizagem adequadas podem se tornar fatores importantes para a explicação dos seus resultados escolares. Além disso, sugere-se que novos trabalhos busquem analisar essa temática por meio de diferentes abordagens de pesquisa, ampliando assim, a investigação sobre as atribuições causais em contexto educacional.

No que se refere à idade e à escolaridade, nessa amostra, verificou-se que os estudantes mais novos e de menor escolaridade fazem mais atribuições causais internas e controláveis ao sucesso escolar obtido, se comparados aos alunos mais velhos e de escolaridade avançada. Esses dados não eram esperados, pois segundo os estudos internacionais e nacionais, com o avançar da idade os alunos tendem a mencionar mais causas internas como fatores responsáveis do sucesso e do fracasso alcançado (Boruchovitch, 2004; Khodayarifard et al., 2006). Entende-se que no início da vida escolar, geralmente, a criança justifica o seu desempenho por meio de explicações menos adaptativas como "Vou mal na escola porque sou burro", "Vou mal na escola porque não tenho sorte" e "Tirei notas boas porque a professora gosta de mim". À medida que avançam na idade e na escolaridade, os alunos começam a perceber que o sucesso e o fracasso dependem da quantidade de esforço aplicado na realização da atividade (Boruchovitch, 2004; Martini \& Boruchovitch, 2004; Miranda et al., 2012). Assim, é interessante a realização de novos estudos com abordagens diferenciadas a fim de coletar informações mais profundas a respeito desse dado interessante e inusitado. É, pois, relevante que se busque compreender porque alunos mais velhos e mais avançados na escolarização estão revelando padrões atribucionais mais disfuncionais e menos adaptativos ao sucesso escolar.

A escola tem um papel essencial na melhoria dos processos atribucionais dos estudantes. Uma das possibilidades interventivas junto aos alunos, especialmente aqueles mais velhos, em anos escolares mais avançados e com histórico de reprovação, são os programas de retreinamento das atribuições de causalidade. De acordo com Boruchovitch e Martini (1997), eles têm o objetivo de mudar como os alunos interpretam suas experiências de sucesso e de fracasso escolar, levando-os a refletirem de forma a substituir as atribuições causais mal adaptativas por aquelas que mais promovem o sucesso escolar. Esses programas atuam nas dimensões da causa, por exemplo, alterando as explicações de fracasso vistas como incontroláveis para controláveis e as externas para internas. Pesquisas mostram que os programas de retreinamento das atribuições de causalidade são promissores em desenvolver no aluno, crenças compatíveis com a motivação para a aprendizagem, maior persistência e consciência de que os eventos podem ser alterados pelo sujeito (Bzuneck \& Sales, 2011; Martini \& Boruchovitch, 2004; Martini \& Del Prette, 2005; Perry, Chipperfield, Hladkyj, Pekrun \& Hamm, 2014; Stewart, Clifton, Daniels, Perry, Chipperfield, \& Ruthig, 2011).

Em linhas gerais, os resultados do presente estudo revelam que, em parte os dados estão de acordo com os resultados encontrados em pesquisas nacionais (Boruchovitch, 2004; Ferreira et al., 2002; Martini \& Boruchovitch, 2009; Martini \& Boruchovitch, 2009; Martini \& Del Prette, 2005). No entanto, alguns achados não corroboraram os pressupostos da Teoria da Atribuição de Causalidade, nem foram consistentes com dados provenientes da literatura brasileira (Boruchovitch, 1994, 2001, 2004; Martini \& Boruchovitch, 2004, 2009; Martini \& Del Prette, 2005; Schilieper, 2001). As incongruências encontradas direcionam a questões referentes ao contexto investigado, visto que os participantes do presente estudo são de uma escola pública situada numa cidade interiorana de pequeno porte. Por um lado, é possível que aspectos relacionados à organização da 
escola, à gestão educacional, a fatores pedagógicos e até mesmo regionais tenham interferido nas crenças e no comportamento dos alunos e produzido resultados diferentes. Por outro, as diferenças obtidas podem também ter sido decorrentes da mensuração das atribuições de causalidade de forma quantitativa com base em um instrumento usado, pela primeira vez, no presente estudo. Assim, acredita-se na relevância de realizar futuras pesquisas orientadas tanto a investigar as atribuições de causalidade, em realidades educacionais diversas à luz de variáveis macro e micro contextuais, como a explorar o impacto que as formas distintas de obtenção de dados possam ter nas atribuições dos estudantes.

\section{CONSIDERAÇÕES FINAIS}

Pode-se dizer que os objetivos do presente estudo foram atingidos. Destaca-se o fato de os alunos apresentarem, predominantemente, causas internas e controláveis como justificativas do sucesso e do fracasso escolar, revelando uma tendência a se responsabilizar pelo seu desempenho. De modo geral, alunos sem repetência atribuíram o sucesso e o fracasso escolar mais a fatores internos e controláveis quando comparados aos estudantes com histórico de reprovação. Não foram encontradas, no entanto, diferenças estatisticamente significante quanto ao sexo na escala de atribuição de causalidade.

Diferentemente do esperado, os alunos de menor idade e escolaridade foram mais propensos a acreditar que o sucesso escolar seja desencadeado por fatores internos e controláveis, quando comparados aos estudantes de maior idade e ano escolar mais avançado. Os dados ora encontrados reforçam a importância de causas atribucionais internas e controláveis tanto para a justificativa do bom como do fraco desempenho escolar. Tais atribuições causais, como por exemplo, a capacidade e o esforço, apresentam-se pertinentes nas situações de sucesso, já que estão associadas a sentimentos de eficácia e levam a motivação para aprender quando o aluno alcança o êxito. No entanto, situação inversa pode ocorrer quando o aluno associa o seu fraco rendimento a baixa capacidade, conforme apresentaram os alunos com repetência escolar, no presente estudo. Esses estudantes necessitam da ajuda dos professores ou profissionais de educação no sentido de reinterpretar sua experiência de fracasso, reatribuindo-na à falta de esforço e/ou ao uso de estratégias de estudo inadequadas. Do mesmo modo, alunos mais velhos e de anos escolares mais avançados também merecem ser alvo de intervenções, pois podem estar desenvolvendo padrões atribucionais mais disfuncionais, à medida que avançam no tempo e na escolarização. Nesse sentido, espera-se que o presente estudo possa lembrar às instituições escolares e aos educadores sobre a importância e a necessidade de promover nos alunos uma reflexão a respeito das causas do resultado escolar alcançado a fim de direcionálos a crenças e comportamentos que estimulem a aprendizagem.

Ademais, o fato de os resultados encontrados sobre as diferenças nas atribuições de causalidade em relação às variáveis sexo, idade e escolaridade não terem ocorrido na direção esperada remete a necessidade de que mais pesquisas sejam conduzidas em regiões pouco estudadas, já que variáveis sociais, culturais, regionais e econômicas, entre outras, podem também interferir nas crenças do aluno. Tais dados nos direcionam também a reflexões a respeito das possíveis diferenças existentes no ensino das escolas brasileiras.

Como limitações da presente pesquisa, compete mencionar, principalmente, aquelas relacionadas ao delineamento metodológico, em função do qual se concentrou esforços em uma única escola, sendo ela pública e localizada numa cidade interiorana de pequeno porte. Dessa forma, os resultados obtidos não são generalizáveis para outras populações. Outra limitação diz respeito ao fato de a Escala de Avaliação das Atribuições de Causalidade para Sucesso e Fracasso Escolar ter sido utilizada pela primeira vez no presente estudo. Os valores dos Alphas de Cronbach obtidos foram, sem dúvida, satisfatórios e aceitáveis (Levin \& Fox, 2004), porém não apresentaram magnitudes altas, o que revela ser interessante um refinamento do instrumento e a sua aplicação em novas amostras, em futuras pesquisas.

De todo modo, apesar de suas limitações, observase que a investigação oportunizou a obtenção de dados que reforçam a importância das crenças atribuicionais do aluno referente ao seu desempenho escolar, gerando maior compreensão das diferenças nas atribuições em razão de variáveis como sexo, idade, repetência e escolaridade, em uma amostra de uma região pouco investigada. Possibilitou também a avaliação e o uso de uma medida objetiva para mensuração dessas atribuições, até então examinadas de forma mais qualitativa, em nosso meio (Boruchovitch, 2004; Martini \& Del Prette, 2005; Marques, De Rose, Del'Arco, \& Martini, 2006; Paiva \& Boruchovitch, 2010; Yoshimoto, 2004). Assim, espera-se que as informações ora obtidas possam ser úteis para os docentes e educadores na identificação dos alunos que precisam de ajuda e contribuam para a promoção da aprendizagem de maior qualidade (Dela Coleta \& Dela Coleta, 2006; Martini \& Boruchovitch, 2009; Weiner, 2013). 


\section{REFERÊNCIAS}

Almeida, L. S. \& Guisande, M. A. (2010). Atribuições Causais na Explicação da Aprendizagem Escolar. In E. Boruchovitch, J. A. Bzuneck, \& S. E. R. Guimarães (Orgs.). Motivação para Aprender - aplicações no contexto educativo. (pp. 145-166). Petrópolis: Vozes.

Almeida, L. S., Miranda, L., \& Guisande, M. A. (2008). Atribuições Causais para o Sucesso e Fracasso Escolares. Estudos em Psicologia, 25(2), 169-176. http://dx.doi.org/10.1590/S0103-166X2008000200001

Almeida, L. S., Miranda, L. C., Salgado, A. M., Silva, M., \& Martins, V. A. (2012). Impacto da Capacidade cognitiva e das atribuições causais no rendimento escolar na Matemática. Quadrante, 21(1), 55-66.

Barreira, S. D. (2010). Teorias Cognitivas da motivação e sua Relação com o Desempenho Escolar. Poíesis Pedagógica, $8(2), 159-175$.

Boruchovitch, E. (1994). As variáveis psicológicas e o processo de aprendizagem: uma contribuição para a Psicologia Escolar. Psicologia: Teoria e Pesquisa, 10(1), 111-128.

Boruchovitch, E. (2001). Conhecendo as Crenças sobre Inteligência, Esforço e Sorte de Alunos Brasileiros em Tarefas Escolares. Psicologia: Reflexão e Crítica, 14(3), 461-467. http://dx.doi.org/10.1590/S0102-79722001000300003

Boruchovitch, E. (2004). A Study of Causal Attributions for Success and Failure in Mathematics Among Brazilian Students. Revista Interamericana de Psicologia, 38(1), 53-60.

Boruchovitch, E. \& Martini, M. L. (1997). As atribuições de causalidade para o sucesso e o fracasso escolar e a motivação para a aprendizagem de crianças brasileiras. Arquivos Brasileiros de Psicologia, 49(3), 59-71.

Boruchovitch, E. \& Santos, A. A. A. (2011). Escala de Avaliação das Atribuições de Causalidade para Sucesso e Fracasso Escolar. Manuscrito não publicado. Universidade Estadual de Campinas, Campinas, SP.

Bzuneck, J. A. \& Sales, K. F. S. (2011). Atribuições interpessoais pelo professor e sua relação com emoções e motivação do aluno. Psico-USF, 16(3), 307-315. http://dx.doi.org/10.1590/S1413-82712011000300007

Cleary, T. J. \& Zimmerman, B. J. (2012). A Cyclical Self-Regulatory Account of Student Engagement: Theoretical Foundations and Applications. In: Handbook of Research on Student Engagement. S. L. Christenson, A. L. Reschly, C. Wylie. (pp. 237-257). New York: Springer US. http://dx.doi.org/10.1007/978-1-4614-2018-7_11

Collares, C. A. L. (1995). O cotidiano escolar patologizado: Espaço de preconceitos e práticas cristalizadas. Tese de Livre Docência. Universidade Estadual de Campinas, São Paulo, Brasil.

Dela Coleta, J. A., \& Dela Coleta, M. F. (2006). Atribuição de causalidade: Teoria, pesquisa e aplicações. (2 $2^{\underline{a}}$ ed.). Taubaté: Cabral Livraria e Editora Universitária.

Fatemi, A. H. \& Asghari, A. (2012). Attribution Theory, Personality Traits, and Gender Differences among EFL Learners. International Journal of Education, 4(2), 181-201. http://dx.doi.org/10.5296/ije.v4i2.1455

Ferreira, M. C., Assmar, E. M. L., Omar, A. G., Delgado, H. U., González, A. T., Silva, J. M. B., Souza, M. A., \& Cisne, M. C. F. (2002). Causal Attribution Success and Falilure in School: a Transcultural Study Brazil-Argentina-Mexico. Psicologia Reflexão e Crítica, 15(3), 515-527. http://dx.doi.org/10.1590/S0102-79722002000300006

Instituto Brasileiro de Geografia e Estatística - IBGE (2014). Acessado em: 30 de outubro de 2014. Disponível em: http://www.ibge.gov.br

Janeiro, I. N. (2011). Escala de Atribuições em relação à Carreira (EAC): um estudo exploratório. Revista Brasileira de Orientacão Profissional, 12(1), 5-13.

Jung, L. M. (2008). Atribuição de causalidade ao insucesso nas provas do simulado do PAS, por alunos e professores do ensino médio: um estudo de caso. Dissertação de Mestrado. Universidade Católica de Brasília, Brasília. Distrito Federal, Brasil.

Khodayarifard, M., Anshel, M. H., \& Brinthaupt, T. M. (2006). Relationships Between Attributional Style and Trait Anxiety for Preadolescent Australian Boys and Girls. Australian Journal of Educational \& Developmental Psychology, 6, 26-38.

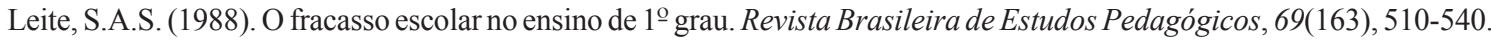

Levin, J. \& Fox, A. J. (2004). Estatística para ciências humanas (9aㅡ ed.). São Paulo: Pearson Prentice Hall.

Marques, T. M. \& Dela Coleta, M. F. (2010). Atribuição de causalidade e reações de mulheres que passaram por episódios de violência conjugal. Temas em Psicologia, 18(1), 205-218.

Martini, M. L. (2003). Variáveis Psicológicas de Professores e Alunos, Ações Interativas e Desempenho Acadêmico: investigando possíveis relações. Tese de Doutorado. Universidade de São Paulo, Ribeirão Preto. São Paulo, Brasil.

Martini, M. L. \& Boruchovitch, E. (2004). A teoria da atribuição da causalidade: contribuições para a formação e atuação de educadores. Campinas: Alínea.

Martini; M. L. \& Boruchovitch, E. (2009). Atribuições de causalidade: a compreensão do sucesso e fracasso escolar por crianças brasileiras. In E. Boruchovitch, A. Bzuneck (Orgs.). A Motivação do Aluno: contribuições da psicologia contemporânea. (pp. 148-166). Rio de Janeiro: Vozes.

Psico, Porto Alegre, v. 46, n. 2, pp. 176-187, abr.-jun. 2015 
Martini; M. L. \& Del Prette, Z. A. P. (2005). Atribuições de Causalidade e Afetividade de Alunos de Alto e Baixo Nível Desempenho Acadêmico em Situações de Sucesso e de Fracasso Escolar. Interamerican Journal of Psychology, $39(3), 355-368$.

Mascarenhas, S., Almeida, L. S., \& Barca, A. (2005). Atribuições Causais e Rendimento Escolar: Impacto das Habilitações Escolares dos Pais e do Gênero dos Alunos. Revista Portuguesa de Educação, 18(1), 77-91.

Miranda, L. C., Almeida, L. S., Boruchovitch, E., Almeida, A. R., \& Abreu, S. A. (2012). Atribuições causais e nível educativo familiar na compreensão do desempenho escolar em alunos portugueses. Psico-USF, 17(1), 1-9. http:// dx.doi.org/10.1590/S1413-82712012000100002

Neves, M. B. J. \& Almeida, S.F.C. (1996). O fracasso escolar na $5^{\underline{a}}$ série, na perspectiva de alunos repetentes, seus pais e professores. Psicologia: Teoria e Pesquisa, 12, 147-156.

Neves, S. P., \& Faria, L. (2007). Auto-eficácia académica e atribuições causais em Português e Matemática. Análise Psicológica, 4(25), 635-652.

Pain, S. (1985). Diagnóstico e tratamento de problemas de aprendizagem. Porto Alegre: Artes Médicas.

Paiva, M. L. M. F. \& Boruchovitch, E. (2010). Orientações Motivacionais, Crenças Educacionais e Desempenho Escolar de Estudantes do Ensino Fundamental. Psicologia em Estudo, 15(2), 381-389. http://dx.doi.org/10.1590/S141373722010000200017

Patto, M. H. S. (1990). A produção do fracasso escolar: histórias de submissão e resistência. São Paulo: TA Queiroz.

Perry, R. P., Chipperfield, J. G., Hladkyj, S., Pekrun, R., \& Hamm, J. M. (2014). Attribution-Based Treatment Interventions in Some Achievement Settings. In Motivational Interventions (pp. 1-35). Emerald Group Publishing Limited. http:// dx.doi.org/10.1108/S0749-742320140000018000

Prieto, G. \& Mu-iz, J. (2000). Um modelo para evaluar la calidad de los tests utilizados em Espa-a. Disponível em: http:// www.cop.es/tests/modelo.htm

Ribeiro, S.C. (1993). A Educação e a inserção do Brasil na modernidade. Cadernos de Pesquisa, 84, 63-82.

Russell, D. (1982). The causal dimension scale: A measure of how individuals perceive causes. Journal of Personality and Social Psychology, 42(6), 1137-1145. http://dx.doi.org/10.1037/0022-3514.42.6.1137

Sá, L. G. C., Dela Coleta, M., \& Dela Coleta, J. A. (2011). Competência Percebida e Atribuição de Causalidade em Jogadores Juniores de Futebol. Psicologia: Teoria e Pesquisa, 27(1), 65-72.

Sahinkarakas, S. (2011). Young students'sucess and failure attributions in language learning. Social Behavior and Personality, 39(7), 879-886. http://dx.doi.org/10.2224/sbp.2011.39.7.879

Schilieper, M. D. M. J. (2001). As Estratégias de Aprendizagem e as Atribuições de Causalidade de Alunos do Ensino Fundamental. Dissertação de Mestrado. Universidade Estadual de Campinas, Campinas. São Paulo, Brasil.

Silva, G. C. R. F., Mascarenhas, S. A. N., \& Silva, I. R. (2011). Vivências de Reprovação e as Atribuições Causais de Estudantes Sobre o Rendimento Escolar em Manaus. In: X Congresso Nacional de Psicologia Escolar e Educacional, Maringá. Disponível em: http://www.abrapee.psc.br/xconpe/trabalhos/1/249.pdf

Soares, J. F. \& Candian, J. F. (2007). O efeito da escola básica brasileira: as evidências do PISA e do SAEB. Revista Contemporânea de Educação, 2(4). Timm, L. A., Argimon, I. I. L., \& Wendt, G. W. Envelhecimento, qualidade de vida e lócus de controle. Perspectiva, 35(129), 131-141.

Soric, I. (2009). Regulatory Styles, Causal Attributions and Academic Achievement. School Psychology International, 30(4), 403-420. http://dx.doi.org/10.1177/0143034309106946

Stewart, T. L. H., Clifton, R. A., Daniels, L. M., Perry, R. P., Chipperfield, J. G., \& Ruthig, J. C. (2011). Attributional retraining: reducing the likelihood of failure. Social Psychology of Education, 14(1), 75-92. http://dx.doi.org/10.1007/ s11218-010-9130-2

Souza, M. P. R. (2009). Psicologia Escolar e Educacional em busca de novas perspectivas. Revista Semestral da Associação Brasileira de Psicologia Escolar e Educacional (ABRAPEE), 13(1), 179-182. http://dx.doi.org/10.1590/ S1413-85572009000100021

Stephanou, G. (2012). Students' School Performance in Language and Mathematics: Effects of Hope on Attributions, Emotions and Performance Expectations. International Journal of Psychological Studies, 4(2), 93-119. http://dx.doi. org/10.5539/ijps.v4n2p93

Weiner, B. (1972). Attribution Theory, Achievement Motivation, and the Educational Process. American Educational Research Association, 42(2), 203-215. http://dx.doi.org/10.3102/00346543042002203

Weiner, B. (1979). A theory of motivation for some classroom experiences. Journal of Educational Psychology, 71(1), 3-25. http://dx.doi.org/10.1037/0022-0663.71.1.3

Weiner, B. (1985). An attributional theory of achievement motivation and emotion. Psycological Rewiew, 92(2), 548-573. http://dx.doi.org/10.1037/0033-295X.92.4.548

Weiner, B. (1991). Metaphors in motivation and attribution. American Psychologist, 46(9), 921-930. http://dx.doi. org/10.1037/0003-066X.46.9.921 
Weiner, B. (2004). Attribution theory revisited: Transforming cultural plurality into theoretical unity. In Research on Sociocultural influences on motivation and learning (pp. 13-29). Information Age Publishing, Inc.

Weiner, B. (2010). The Development of an Attribution-Based Theory of Motivation: A History of Ideas. Educational Psychologist, 45(1), 28-36. http://dx.doi.org/10.1080/00461520903433596

Weiner, B. (2013). An Attributional Theory of Behavior. In B. Weiner (Org.). Human motivation. Psychology Press.

Yoshimoto, C. R. (2004). Análise atribucional do baixo rendimento escolar em alunos da quarta série do ensino fundamental, participantes do projeto "Escola nas Férias". Dissertação de Mestrado. Universidade Estadual Paulista, Marília. São Paulo, Brasil.

Autores:

NatÁlia Rodovalho Garcia - Doutorando, Universidade de São Paulo.

EVELY BORUCHOVITCH - Universidade Estadual de Campinas.

Endereço para correspondência:

Natália Rodovalho Garcia

Rua Afonso Celso, 171 - Bloco A, Ap. 94 - Vila Mariana

CEP 04119-000 São Paulo, SP, Brasil

E-mail: nataliarodovalhogarcia@yahoo.com

Recebido em: 07.06.14

Aceito em: 10.03.15 